\title{
Politique
}

\section{L'élection fédérale de 1988 et la défaillance du NPD}

\section{André Lamoureux}

Numéro 15, hiver 1989

Paradigmes et scientificité

URI : https://id.erudit.org/iderudit/040620ar

DOI : https://doi.org/10.7202/040620ar

Aller au sommaire du numéro

Éditeur(s)

Société québécoise de science politique

ISSN

0711-608X (imprimé)

1918-6584 (numérique)

Découvrir la revue

Citer cet article

Lamoureux, A. (1989). L'élection fédérale de 1988 et la défaillance du NPD.

Politique, (15), 79-103. https://doi.org/10.7202/040620ar d'utilisation que vous pouvez consulter en ligne.

https://apropos.erudit.org/fr/usagers/politique-dutilisation/ 


\title{
L'élection fédérale de 1988 et la défaillance du NPD
}

\author{
André Lamoureux \\ Cegep Andre-Laurendeau
}

La présente étude, traitant du NPD, constitue un complément d'analyse à celle parue dans le numéro 14 de la revue Politique. Elle examine la portée des élections du 21 novembre 1988 pour le NPD et suggère quelques axes d'analyse et de recherche.

En prévision des élections fédérales du 21 novembre 1988, les dirigeants du Nouveau Parti démocratique, tant ceux du parti fédéral que ceux de l'aile québécoise, ont escompté un renforcement sensible des positions du NPD à l'échelle pan-canadienne. Bien qu'une baisse des appuis populaires du NPD ait été constatée dans les sondages au cours de l'année 1988 (en comparaison avec les sommets atteints en 1987), les intentions de vote en faveur de ce parti sont restées élevées durant la prériode pré-électorale. Le NPD a cru pouvoir ainsi capitaliser. Cette expectative du NPD s'appuyait surtout sur l'espoir qu'il entretenait de réaliser enfin une «percée historique» au Québec et d'y faire élire, pour 
la première fois de son histoire, un ou quelques députés. Ce qui ne s'est pas réalisé.

La portée de cette élection fédérale de 1988 pour le NPD revêt une signification toute particulière. Survenant 30 ans après le coup d'envoi donné à la fondation du «Nouveau Parti» par le Congrès du travail du Canada (avril 1958), offrant au NPD une occasion comme il ne s'en est pas présentée après sa fondation, et faisant suite à une série d'échecs répétés au Québec, l'élection fédérale du 21 novembre 1988 dépasse en importance, pour le NPD, toutes les élections fédérales antérieures auxquelles il lui a été donné de participer.

Pour ces raisons, cet épisode majeur de l'évolution récente du NPD mérite un complément d'analyse. Le présent texte, loin de prétendre donner une explication exhaustive de l'élection fédérale de 1988, soumet des propositions d'explication et des pistes de recherche quant au sort dévolu au NPD. L'objectif étant de mieux comprendre les difficultés et les obstacles de la social-démocratie au Canada depuis les années 1960, notamment en relation avec la question nationale québécoise.

\section{Les résultats de l'élection fédérale de 1988}

L'analyse de la performance du NPD dans l'élection fédérale du 21 novembre 1988 doit d'abord prendre en considération les résultats de cette élection tout comme la campagne électorale qui l'a précédée, cette dernière ayant été particulièrement dominée par la question du libreéchange. Le sens de cette élection doit être délimité car il conditionne immanquablement les conclusions que l'on peut tirer de la défaillance qu'y a manifestée le NPD.

Au plan des faits, rappelons que l'élection du 21 novembre 1988 a permis au Parti conservateur de renouveler sa victoire de 1984 . Elle lui a aussi permis d'obtenir, pour une deuxième fois consécutive, une majorité absolue de sièges, ce qu'aucun parti fédéral n'était parvenu à réaliser après 1953. Le parti de Brian Mulroney a ainsi remporté 169 
sièges, contre 83 pour le Parti libéral et 43 pour le NPD ${ }^{1}$. Le gouvernement conservateur s'est donc assuré d'une majorité confortable, même si celle-ci était réduite par rapport à 1984.

Cette victoire a été suivie d'analyses diverses, souvent réductrices. On a souvent expliqué qu'en vertu de la majorité de sièges recueillis par les conservateurs, la population aurait, par le fait même, tranché en faveur du libre-échange ${ }^{2}$, ce qui expliquerait la défaite et du Parti libéral et du NPD.

Cependant, le 21 novembre 1988 ne fait pas exception aux autres élections. L'analyse des résultats de cette élection fédérale exige une mise en situation et un examen rigoureux des voix recueillies par chaque parti au suffrage populaire, sans oublier les abstentions. Seuls ces éléments nous permettent d'apprécier le partage des options politiques exprimées le 21 novembre 1988 et le niveau d'appui populaire réel des partis, dont le NPD. En l'occurrence, le seul nombre de sièges obtenus par les partis reste toujours, dans ce cas comme dans les autres, le reflet déformé de la réalité politique ${ }^{3}$.

En fait, sans même considérer la part des abstentions (26\%), il ressort que le Parti conservateur a recueilli $43 \%$ des suffrages exprimés au plan canadien, soit effectivement une minorité des voix. Le 21 novembre 1988, une majorité de votants s'est prononcée contre le gouvernement Mulroney. Ensemble, en effet, le Parti libéral et le NPD ont obtenu $52,3 \%$ des voix, selon les données d'ÉlectionsCanada. Le PLC a recueilli $31,9 \%$ des voix exprimées et le NPD $20,4 \%$. Les autres petits partis et les candidats indépendants, tous ensemble, ont réuni quelque $4,7 \%$ des voix. Ce seul constat, quant au partage des voix, contredit l'affirmation selon laquelle le gouvernement Mulroney aurait obtenu, avec ce scrutin, un appui de la population

1. Les données à propos de cette élection sont tirées du rapport préliminaire du Directeur général des élections, février 1989.

2. C'est une opinion qui a été exprimée chez Brian Mulroney et son équipe, dans le monde des affaires et les associations patronales, et parmi les commentateurs de la presse écrite, de la radio et de la télévision. Ce fut même l'opinion d'Ed Broadbent au lendemain de l'élection (Le Devoir, 23 novembre 1988).

3. Les élections québécoise de 1944, de 1966, de 1970 ou de 1973 en sont quelques exemples très révélateurs. 
canadienne sur le projet de libre-échange qu'il pilotait. Pareil énoncé, pour être crédible, même en écartant les abstentions, aurait exigé tout au moins que le gouvernement bénéficie d'une majorité parmi les voix exprimées.

Qu'est-ce à dire? Le vote majoritaire qu'ont pu recueillir ensemble le Parti libéral et le NPD le 21 novembre signifiait-il pour autant un vote d'opposition au libre-échange, du moins un vote d'opposition au projet de traité Reagan-Mulroney soumis par les conservateurs? Bien que les évaluations à ce propos soient restées controversées, nous suggérons pour notre part qu'au plan canadien le décompte des voix exprimées dans cette élection fédérale contient un tel message, et ce malgré la victoire conservatrice. Ce diagnostic s'appuie, d'une part, sur le fait que, durant toute la campagne électorale, les libéraux ont officiellement lié le vote libéral à un vote d'opposition au traité proposé par les conservateurs (indépendamment de ce qu'ils auraient fait par la suite). D'autre part, le vote en faveur du NPD, était tout autant associé à un vote d'opposition au dit projet de libre-échange. En d'autres mots, le vote du 21 novembre 1988, sur un plan d'ensemble, ne peut être interprété comme un vote pro-libreéchange. Les bases de la victoire conservatrice doivent être mieux situées.

Dans cette optique, il faut noter que le Parti conservateur n'a pu récolter une majorité de voix que dans deux seules provinces: en Alberta (52\% des voix exprimées) et au Québec (53\%). Certes, dans ces deux cas, le Parti conservateur a drainé une majorité des voix derrière ses positions. Victoires significatives, voire éclatantes. Mais il reste que ce sont bel et bien ces deux provinces, l'Alberta et le Québec, qui ont permis aux conservateurs de remporter une majorité de sièges et de sortir gagnant de l'élection au plan canadien. Dans le reste du Canada, la bataille s'est avérée beaucoup plus serrée (voir le tableau 1). De plus, comme la victoire conservatrice était acquise d'avance en Alberta $^{4}$ et que seulement 26 sièges y étaient en jeu (dont un seul a

4. Il s'agit d'une forteresse conservatrice, malgré les récentes percées du NPD. Toutefois, en Alberta, les conservateurs ont dû faire face à l'émergence d'un nouveau parti d'extrême-droite, le Reform Party. Ce demier a réussi à recueillir $15,4 \%$ des voix. 
échappé au PC), on doit donc conclure que la bataille électorale s'est finalement décidée au Québec. Là les conservateurs y ont remporté une nette majorité des voix exprimées et 63 des 75 sièges $^{5}$.

Tableau 1: Les élections fédérales du 21 novembre 1988. Pourcentage des voix et sièges obtenus par les trois grands partis

\begin{tabular}{|c|c|c|c|c|c|c|c|c|}
\hline \multirow[b]{2}{*}{ Québec } & \multicolumn{2}{|c|}{$\begin{array}{c}\text { voix sièges } \\
\varnothing\end{array}$} & $\underset{\%}{\text { voix }}$ & $\begin{array}{l}\text { LC } \\
\text { sièges }\end{array}$ & \multicolumn{2}{|c|}{$\begin{array}{l}\text { NPD } \\
\text { voix sièges } \\
\%\end{array}$} & \multicolumn{2}{|c|}{$\begin{array}{l}\text { Autres } \\
\text { voix sièges } \\
\%\end{array}$} \\
\hline & 52,7 & 63 & 30,3 & 12 & 14 & 0 & 3,1 & 0 \\
\hline Ontario & 38,2 & 46 & 38,9 & 43 & 20,1 & 10 & 2,9 & $\mathbf{0}$ \\
\hline I.-P.E. & 41,5 & 0 & 49,9 & 4 & 7,5 & 0 & 1,1 & 0 \\
\hline N.-Brunswick & 40,4 & 5 & 45,4 & 5 & 9,3 & 0 & 4,9 & 0 \\
\hline N.-Écosse & 40,9 & 5 & 46,5 & 6 & 11,4 & 0 & 1,2 & 0 \\
\hline Terre-Neuve & 42,2 & 2 & 45,0 & 5 & 12,4 & 0 & 0,4 & 0 \\
\hline Manitoba & 36,9 & 7 & 36,5 & 5 & 21,3 & 2 & 5,3 & 0 \\
\hline Saskatchewan & 36,4 & 4 & 18,2 & $\mathbf{0}$ & 44,2 & 10 & 1,2 & 0 \\
\hline Alberta & 51,8 & 25 & 13,7 & 0 & 17,4 & 1 & $17,2 *$ & 0 \\
\hline C.-Britannique & 35,3 & 12 & 20,4 & 1 & 37,0 & 19 & 7,4 & 0 \\
\hline T.N.O. & 26,4 & 0 & 41,4 & 2 & 28,3 & 0 & 3,9 & 0 \\
\hline Yukon & 35,3 & 0 & 11,7 & 0 & 51,4 & 1 & 2,0 & 0 \\
\hline CANADA & 43,0 & 170 & 31,9 & 83 & 20,4 & 43 & 4,7 & 0 \\
\hline
\end{tabular}

Taux de participation aux élections: 74\%. Les pourcentages des partis sont calculés en fonction des votes valides déposés.

* Dont 15,4\% au Reform Party.

Source: Rapport préliminaire d'Élections-Canada, février 1989.

5. Il faut souligner cependant qu'en territoire québécois les voix d'opposition ont quand même totalisé $47 \%$ des suffrages, dont $30 \%$ au PLC, $14 \%$ au NPD et $3 \%$ aux autres partis. Ainsi, au Québec, presqu'une personne sur deux a donné son vote à un parti autre que les conservateurs. 


\section{La victoire conservatrice et le Québec}

Tout compte fait, dans la conquête d'une majorité conservatrice à la Chambre des communes, c'est le Québec qui aura été décisif. C'est au Québec, sa base traditionnelle d'appui, que le Parti libéral a une nouvelle fois échoué. C'est aussi le Québec qui n'a pas non plus accordé au NPD l'ouverture que ce parti escomptait, ce qui explique dans une bonne mesure sa faible performance au plan canadien. Le Québec, bien plus que les Maritimes et l'Ontario, aura été la plaque tournante de cette élection pour tous les partis: les conservateurs, les libéraux, le NPD.

En conséquence, toute analyse de la défaillance du NPD dans l'élection fédérale de 1988 exige que soient d'abord scrutées les conditions de la victoire conservatrice, notamment au Québec.

Dans le partage des voix et des sièges obtenus par les différents partis à cette élection de 1988, plusieurs facteurs différenciés ont joué. La situation particulière de chacune des provinces a également influencé le cours des événements. Mais plus particulièrement en territoire québécois, notre proposition suggère que deux facteurs principaux ont influencé la victoire conservatrice, elle qui a scelle l'issue de l'élection au plan canadien. D'une part, l'impact de la coalition en faveur du libre-échange qui s'est soudée autour du Parti conservateur. Au Québec même, elle a connu un déploiement significatif. D'autre part, l'impact, que nous estimons central, de la question nationale québécoise.

Sous un premier volet, situons l'influence de la coalition prolibre-échange, surtout en territoire québécois.

De manière générale, jusqu'au fameux débat des chefs des 24 et 25 octobre, il n'y avait que très peu de crainte dans le camp conservateur sur l'issue du vote au plan canadien. Les sondages, qui avaient témoigné d'une remontée des conservateurs en 1988, donnaient une bonne longueur d'avance au parti de Brian Mulroney ${ }^{6}$. Les libéraux

6. Un sondage de la maison Angus Reid tenu les 2 et 3 octobre 1988, à l'ouverture de la campagne électorale, attribuait $45 \%$ des intentions de votre au PC, contre $27 \%$ pour le NPD et $26 \%$ pour le PLC. Du 2 au 10 octobre, un autre sondage d'Environics Research donnait 
semblaient s'enliser. Du côté conservateur, on estimait, grosso modo, que la victoire était acquise. Mais peu à peu, notamment suite aux débats des chefs, alors que les sondages indiquaient que l'opposition au libre-échange prenait effectivement de l'ampleur et favorisait la remontée des libéraux ${ }^{7}$, il s'est structuré une importante coalition dans le monde des affaires en faveur du libre-échange.

C'est précisément au Québec que cette coalition s'est manifestée avec le plus d'éclat et avec le plus d'unité. De l'intervention du président de l'Alcan, David Culver, à la constitution du Regroupement pour le libre-échange ${ }^{8}$, l'ampleur qu'a prise cette alliance a peu ou pas de précédent dans l'histoire politique du Québec. Les grandes corporations, les grandes banques, les associations patronales, le Parti conservateur, le PLQ et le PQ ont été de la partie. Ils ont été flanqués des Robert Bourassa, Jacques Parizeau, Bernard Landry, Jean Doré, de plusieurs économistes, et de nombre de porte-parole du monde des affaires (Laurent Beaudoin, Claude Castonguay, Paul Desmarais, Pierre Lortie, Bernard Lamarre, etc.). Toutes ces forces et personnalités se sont circonstanciellement unies pour organiser la riposte et permettre la victoire de Brian Mulroney. Les grandes corporations qui, hier, s'étaient déjà unies dans la coalition Pro-Canada pour assurer la victoire du NON au référendum de 1980 , se sont rassemblées une nouvelle fois. Cette fois-ci, elles ont martelé l'opinion publique pour sauver le projet de libre-échange. Mais à la différence de 1980, l'aile nationaliste du patronat québécois s'est ralliée à la cause. Rarement a-ton vu la classe capitaliste au Québec faire une telle unité au Québec et agir politiquement avec une telle concertation. Les médias québécois,

$42 \%$ au PC, $29 \%$ au NPD et $25 \%$ au PLC. $\AA$ ce moment-là, une importante erreur d'Ed Broadbent fut de sous-estimer la force libérale.

7. Le 7 novembre, un sondage Gallup témoignait du revirement momentané: les libéraux recueillaient $43 \%$ des intentions de vote, le PC $31 \%$ et le NPD $22 \%$. Déjà pour le NPD, c'était un affaissement important de ses appuis.

8. Ce Regroupement pour le libre-échange a été créé au Québec au début du mois de novembre 1988 . Ses activités publiques ont commencé les 9 et 10 novembre, recevant l'appui de plusieurs corporations: Bombardier, la Corporation financière Power, le groupe Canam Manac, etc., et des principales associations patronales du Québec. 
favorables pour la plupart au libre-échange, ont été aussi de la partie. Le cas de La Presse et du Devoir en cours de campagne en ont été les exemples les plus frappants. Tout particulièrement dans la seconde partie de la campagne électorale, l'information y a été soumise très souvent à un traitement purement partisan'.

Ces interventions, d'origines diverses, ont connu un fort déploiement. Les prises de positions répétées de l'élite économique et politique du Québec et de ses personnages les plus en vue, dont Robert Bourassa et Jacques Parizeau, ne pouvaient rester sans effet. On a pu constater, à l'inverse, l'influence qu'a pu exercer David Peterson en Ontario. Dès le 10 novembre, les sondages témoignaient d'ailleurs d'un nouveau renversement des tendances. Les libéraux accusaient désormais une nouvelle baisse, particulièrement au Québec ${ }^{10}$. Sans nullement prétendre que les Québécois ont finalement voté majoritairement en faveur des conservateurs sous l'unique influence de cette coalition prolibre-échange, notre point de vue est à l'effet que celle-ci a pu exercer, notamment au Québec, un rôle non négligeable sur l'opinion et le vote

9. Une analyse du Devoir et de La Presse, du 25 octobre et 21 novembre 1988, nous permet de constater cette orientation. Non seulement dans les pages éditoriales, ce qui va de soi, mais surtout dans le choix des contributions et des points de vue retenus. En l'occurrence, rares ont été les contributions contre l'accord de libre-échange publiées par ces quotidiens, comme celle de Pierre Paquette (président du Conseil central de Montréal - CSN), parue le 22 octobre dans le journal $\mathbf{L a}$ Presse. En cours de campagne et après le 21 novembre 1988, ce fait a été noté par quelques observateurs, dont Georges Mathews, professeur et économiste à l'INRS, comparant même le joumal La Presse à un «instrument de matraquage de l'opinion publique» dans les demiers jours de campagne ( $L a$ Presse, 18 octobre 1988). Par ailleurs, après l'élection, on sait que Marcel Pépin démissionnait précisément du Conseil d'administration du Devoir en signe de réprobation face à l'orientation donnée au journal en cours de campagne.

10. Voir le sondage Angus Reid réalisé pour Le Soleil le 10 novembre 1988. Ce sondage attribuait désormais aux conservateurs $49 \%$ des intentions de vote au Québec, signe d'une nette remontée, alors que le sondage Gallup effectué du 2 au 5 novembre ne leur donnait plus que $32 \%$. 
final. Il reste toutefois bien sûr difficile de quantifier l'ampleur de cette influence.

Sous un autre volet, au Québec, les conservateurs ont aussi misé fortement sur la carte du nationalisme pour s'assurer la victoire, comme ils l'avaient fait en 1984. Mais en 1988, suite aux multiples scandales connus, les conservateurs ont eu un besoin encore plus pressant du Québec. Déjà, au printemps 1988, en propulsant Lucien Bouchard au cabinet, lui qui avait appelé à voter OUI au référendum québécois de 1980, la stratégie de Brian Mulroney se dessinait avec clarté, à savoir le recours au nationalisme. L'élection partielle dans le comté de Lac-Saint-Jean, en juin 1988, a constitué un autre moment clé de cette stratégie. Pendant la campagne électorale, devant le rebondissement des libéraux, Brian Mulroney n'a pas changé de cap. Il n'a pas manqué de rappeler le passé des libéraux au Québec, dont l'humiliation constitutionnelle de 1982 . Les références à l'endroit des Chrétien et des Ouellet ont été multipliées. Brian Mulroney a aussi encore tablé sur sa provenance québécoise et francophone, se présentant comme le défenseur du Québec, en particulier en référence avec l'Accord du Lac Meech. Il a aussi misé sur l'image populiste bien connu du «p'tit gars de Baie Comeau», image par ailleurs aussi utilisée au Canada anglais. Secondé par les Lucien et Benoît Bouchard, le chef conservateur a donc utilisé à fond la ferveur nationaliste et l'a retournée contre les libéraux. Les dirigeants du Parti québécois ont apporté leur concours, dont Jacques Parizeau et Bemard Landry qui ont multiplié les interventions publiques pour défendre le projet de libre-échange et soutenir par le fait même les conservateurs. En définitive, on a ainsi travaillé à créer l'impression selon laquelle le mouvement d'appui aux conservateurs et au libre-échange procédait d'un «bloc québécois»"11.

Cette autre dimension de l'orientation stratégique de la campagne conservatrice au Québec dans le dernier sprint électoral a porté fruit et son effet s'est réflété dans le scrutin final. En témoignent les résultats même de l'élection, tout particulièrement chez les francophones. Dans la région de Montréal, chez les francophones, les

11. L'opposition au libre-échange, comme devait le laisser entendre Lucien Bouchard, fut même associée à une bataille de l'Ontario contre le Québec(!). 
conservateurs ont recueilli davantage de support électoral qu'en 1984. Dans le reste du Québec, l'avance de 1984 s'est aussi affirmée. Dans la région du Saguenay et du Lac-Saint-Jean, par exemple, les conservateurs ont effectué un véritable balayage.

Tout compte fait, nous estimons que l'impact de la forte coalition en faveur du libre-échange ainsi que le recours à l'arme puissante du nationalisme se sont conjugués pour devenir les deux principaux atouts des conservateurs au Québec dans cette victoire du 21 novembre 1988.

Dans une telle situation, le sort réservé au Parti libéral et au NPD n'en est que mieux éclairé. Dans les deux cas, estimons-nous, les résultats obtenus par ces partis ont également été fonction, dans une large mesure, des deux enjeux mentionnés, le libre-échange et la question nationale québécoise.

\section{La nouvelle défaite des libéraux}

Dans le cas des libéraux, ceux-ci ont bien sûr réussi, au plan canadien, à canaliser la plus grande part des voix d'opposition et, par rapport à 1984, à plus que doubler le nombre de leur députation. Les libéraux ont obtenu un vote particulièrement important dans les Maritimes, mais aussi en Ontario (39\% des voix contre 38\% pour les conservateurs) et au Manitoba. Ce résultat est venu prolonger, au plan fédéral, les gains importants qu'ils ont réalisés dans ces provinces au cours de l'année 1987 et celle de $1988^{12}$. Malgré les hésitations et les incertitudes manifestées en début de campagne envers leur chef fédéral, les David Peterson, Frank McKenna et Sharon Carstairs ont finalement déployé tous leurs efforts dans la bataille électorale. Au Manitoba et en Ontario, les forces libérales ont mené une dure lutte au libre-échange. Elles ont clairement menacé les conservateurs, et ont simultanément affaibli le vote en faveur du NPD. Le Parti libéral s'est

12. À propos du renforcement des assises libérales dans ces provinces en 1987 et 1988, voir l'analyse que nous avons effectuée dans le précédent numéro de la revue Politique (14), p. 102-104. 
donc partiellement relevé, dans quelques provinces, de la défaite subie en 1984.

Cependant, ce ne fut pas le cas au Québec. Les libéraux n'ont pas été en mesure d'y effectuer une remontée. Au contraire, de 1984 à 1988, leurs appuis ont encore chuté. Ils sont passés de $35,4 \%$ du vote populaire à 30\%. La faiblesse du vote libéral en 1988 s'explique donc, dans une large mesure, par les résultats obtenus au Québec.

Pour évaluer cette ratée libérale au Québec, il faut se souvenir que l'élection fédérale de 1984 a été le thêâtre d'un fort mouvement de rejet. Cette élection a entraîné une défaite sans précédent pour les libéraux, notamment chez la population francophone. Le peuple québécois s'est alors tourné massivement contre ce parti. Non seulement y avait-il un contexte de récession, marqué par les traces d'une politique monétaire douloureuse et par une hausse du taux de chômage; non seulement le parti était-il affaibli par le leadership de John Turner et la division interne du parti, ou encore par le fait que John Turner était un chef anglophone mal reçu au Québec. Ces éléments d'explication, et d'autres, sont importants. Mais ils ne suffisent pas. Il faut aussi souligner que cette élection survenait dans la foulée du Coup de force constitutionnel de 1982 et de l'attitude manifestée par les libéraux contre les aspirations nationales du peuple québécois, notamment sur le plan linguistique. Cette dernière dimension de l'élection de 1984 a généralement été sous-estimée dans les analyses qui l'ont suivie. Pourtant elle a été reflétée par le vote luimême, au premier chef dans les comtés à prépondérance francophone, notamment à l'extérieur de Montréal. En effet, en 1984, à l'exception de quatre circonscriptions, les comtés situés hors de Montréal, pour la plupart très francophones, sont passés du côté conservateur.

L'élection de 1988 semble démontrer que la réalité nationale québécoise, en plus du libre-échange, a joué à nouveau dans le résultat. Même si John Turner s'est prononcé contre le libre-échange et a joué la carte du populisme pour remonter sa cote de popularité et gagner l'appui des classes populaires, il reste que la majorité des Québécois qui ont voté, tout particulièrement chez les francophones, ont encore une fois refusé de voter libéral et se sont exprimés en faveur de Brian Mulroney. D'ailleurs, la baisse globale du vote libéral au Québec en 1988 s'explique d'abord et avant tout par la diminution des appuis 
recueillis chez les francophones, notamment sur l'Ile de Montréal $(24,9 \% \text { contre } 27,9 \% \text { en } 1984)^{13}$. À une exception près, soit le comté de Sainte-Marie remporté par Jean-Claude Malépart, les comtés où les libéraux ont gagné, dans la région de Montréal comme dans les trois situés à l'extérieur de Montréal, sont des comtés à majorité anglophone et allophone (NDG, Mont-Royal...) ou à forte minorité anglophone et allophone (Papineau-Saint-Michel, LaSalle-Émard, ...). Congédiés en 1984, adversaires de la loi 101, partisans de la centralisation des pouvoirs, opposés chez un bon nombre d'entre eux à la reconnaissance du caractère distinct du Québec, les libéraux sont donc encore loin de la reprise en mains au Québec. La division linguistique du vote au Québec indique, à notre avis, que la question nationale a encore joué de tout son poids dans cette nouvelle défaite libérale ${ }^{14}$.

Mais le NPD n'a pas échappé non plus à ce dilemme québécois. L'épreuve a été pour lui encore plus difficile.

\section{La défaillance du NPD}

Le 21 novembre 1988, au plan canadien, le NPD a quant à lui recueilli $20,4 \%$ des voix et a réussi à faire élire 43 députés. En comparaison avec 1984, il s'agit d'une certaine augmentation du nombre de députés élus (13 de plus). Mais il s'agit d'une très faible augmentation du pourcentage de voix recueillies (1,6\% de plus). Cette

13. Pour plus de détails sur le partage des votes dans l'élection du 21 novembre 1988 entre francophones, allophones et anglophones, voir l'étude publiée par Robert Boily, Alain Pelletier et Pierre Serré dans Le Devoir des 8 et 9 décembre 1988 sous le titre «Le pluralisme ethnique et les élections fédérales».

14. La manifestation du MQF d'avril 1988, qui a rassemblé quelque 25,000 personnes en faveur de la loi 101, et les événements qui, par la suite, ont suivi le jugement de la Cour suprême sur l'affichage au Québec confirmaient déjà qu'en cette fin de 1988 les aspirations nationales du peuple québécois, au plan linguistique notamment, demeuraient toujours présentes. Après l'élection, la manifestation du 12 mars 1989, réunissant environ 80,000 personnes en faveur de la loi 101 , a reconfirmé ce fait. 
double facette de la piètre performance réalisée par le NPD, contraire à ses propres attentes, exige que soient mis en lumière les éléments explicatifs de ce résultat.

À première vue, le fait que le NPD ait gagné 43 sièges dans cette élection fédérale, un sommet dans son histoire, peut apparaître comme une réalisation exceptionnelle pour ce parti. Cependant la répartition des sièges et des voix obtenues par le NPD en dit long sur la portée régionale, et donc limitée, de ces gains. En effet, la grande majorité des députés néo-démocrates élus (29 sur 43) l'ont été dans deux provinces seulement: la Colombie-Britannique et la Saskatchewan. Dans ces deux provinces, le renforcement des positions du NPD était prévisible et le NPD y a aussi dépassé ses adversaires quant aux voix recueillies. Dix autres députés ont été fournis par l'Ontario, un par le Yukon, et finalement, deux par le Manitoba et un par l'Alberta.

La Colombie-Britannique, à elle seule, a fourni presque la moitié de la nouvelle députation du NPD. Le pourcentage des voix exprimées en faveur du NPD a aussi atteint un sommet dans cette province pour une élection fédérale (37\%). Ce résultat de la ColombieBritannique, ne peut trouver son explication en ne considérant que les seuls enjeux connus de la camp. gne électorale fédérale. Bien sûr, l'opposition au libre-échange s'est exprimée avec force dans cette province. Elle s'est manifestée non seulement dans les bastions traditionnels du NPD (le mouvement syndical, les travailleurs du bois, les enseignants, etc.), mais aussi dans d'autres couches de la population, dont les agriculteurs de la province. Cette dimension fournit une des données explicatives de l'élargissement des appuis du NPD. Ce mouvement d'opposition au libre-échange a d'ailleurs été dirigé par le NPD, appuyé par de fortes candidatures, dont Michael Hartcourt, chef du parti et ancien maire de Vancouver, et Dave Barrett, ancien premier ministre de la province. Par contre, les résultats du NPD en Colombie-Britannique peuvent aussi être partiellement attribués au fort mécontentement qui, à compter de 1987, s'est développé contre le gouvernement de Bill Vander Zalm (lui-même partisan du libre-échange et solidaire des conservateurs). L'orientation ultra-conservatrice de ce gouvernement a généré cette insatisfaction. À ce titre, on doit souligner la politique de privatisation des services qu'a 
pilotée Vander Zalm ou la remise en cause de législations et de programmes sociaux, dont l'introduction de l'Industrial Relations Reform Act (loi 19) qui a entraîné la grève générale de 350,000 travailleurs (la première à survenir depuis 1913) ${ }^{15}$. Ce mécontentement, estimons-nous, s'est jumelé à la campagne électorale fédérale. Il a permis au NPD d'atteindre une performance qu'il n'a jamais pu réaliser dans cette province à l'occasion d'une élection fédérale.

Quant à la Saskatchewan, le vote néo-démocrate confirme la remontée du NPD qui s'y est dessinée depuis 1986. Le NPD a vraisemblablement profité de ce contexte. Il a aussi tiré profit de sa campagne contre le libre-échange et des difficultés du gouvernement Devine qui, notamment avec son programme de réduction du déficit provincial et de coupures budgétaires, a dû faire face à de plus en plus de réprobation. Au Yukon, la députée Audrey McLaughlin, partisane de la réouverture de l'Accord du Lac Meech dans le but d'octroyer au Yukon le statut de province, a également reçu un fort appui de la population et a renouvelé sa victoire de 1987. En Alberta, le NPD a effectué une première percée dans Edmondon est. Ce gain s'inscrit comme prolongement des progrès effectués lors de l'élection provinciale de 1986.

Mais là s'arrêtent les succès du NPD. Au Manitoba, le mouvement à la baisse, en sièges et en voix, déjà indiqué lors de l'élection fédérale de 1984, a poursuivi sa trajectoire. En Ontario, la percée de 1984 s'est érodée. Supplanté par les conservateurs et les libéraux, le NPD y a été tenu en échec. Tout comme au Manitoba, le NPD a été condamné à un rôle de second plan. Dans ces deux provinces, le NPD s'est surtout avéré incapable de canaliser l'important mouvement d'opposition aux conservateurs qui s'y est exprimé, notamment vis-à-vis du libre-échange. Les libéraux ont littéralement damé le pion aux néo-démocrates. Sharon Carstairs au Manitoba et David Peterson en Ontario ont contribué grandement à ces résultats. Pour ce qui est des provinces maritimes, le NPD y a été contenu à un

15. Pour plus d'informations à ce sujet, voir l'article de Tom Fawkes intitulé «La loi 19: comment en sommes-nous arrivés là?», Le Monde syndical (revue du Conseil du travail du Canada), numéro d'hiver 1988 , p. 7-10. 
rôle marginal ${ }^{16}$. Quant au Québec, le NPD a bel et bien raté la percée qu'il escomptait y réaliser. Nous en traitons dans la dernière partie de cet article.

L'analyse des résultats du NPD en Ontario et au Manitoba permet néanmoins de mettre en évidence ici ce qui nous apparaît être un des principaux facteurs explicatifs de la faible performance du NPD à l'échelle canadienne. Cet élément a également joué dans le cas du Québec, bien que d'autres éléments doivent être considérés.

Bien sûr, une part de l'électorat canadien ne soutient pas le NPD à cause de son programme social ou économique, jugé dépassé par les uns ou trop radical par les autres. Mais une des grandes carences du NPD dans la campagne électorale de 1988 , comme dans la période qui l'a précédée, renvoit à la faiblesse de leadership dont a fait preuve la direction du NPD.

En d'autres mots, le NPD n'aurait pas mené une bataille électorale convaincante lui permettant d'amasser les votes, ratant ainsi l'occasion privilégiée qui lui était offerte.

Plusieurs faits, en effet, tendent à soutenir le point de vue selon lequel le NPD a cédé le pas aux conservateurs et aux libéraux à l'approche du scrutin ${ }^{17}$.

D'une part, un peu comme l'avait fait Bob Rae en Ontario en 1987, il ressort que le chef du NPD a concédé la victoire aux conservateurs dès le début de la campagne. Dès le départ, en effet, Ed Broadbent a évoqué la possibilité de constituer un gouvernement de coalition avec les libéraux, voire même avec les conservateurs, et ce alors même que le NPD était au meilleur de sa cote et semblait

16. Nous n'analysons pas ici la problématique du NPD dans les Maritimes, question qui reste d'ailleurs très peu scrutée dans les études existantes sur le NPD.

17. Ce point de vue a notamment été soutenu par Bob White, Vice-président national du parti et Président du Syndicat canadien des travailleurs de l'automobile. Celui-ci, après l'élection, disait ne jamais avoir vu un tel niveau de déception et de colère chez les militants et les candidats du NPD comme chez les dirigenats du mouvement syndical. Pierre Graveline, alors co-président du NPD-Québec, a aussi exprimé publiquement un point de vue semblable (Le Devoir, ler décembre 1988). 
pouvoir se substituer au PLC comme deuxième parti au Canada. Cette stratégie devenait guère encourageante pour ceux et celles qui comptaient voter NPD pour battre les conservateurs, notamment en fonction du libre-échange. Le NPD a donc mené une campagne où, dès les premiers instants, il s'affichait perdant. Ce qui ne pouvait que profiter aux libéraux.

Aussi, alors que le NPD avait été le premier parti à exiger une élection sur le libre-échange, l'initiative de l'opposition au traité Reagan-Mulroney a été rapidement concédée aux libéraux. Les stratèges néo-démocrates ont orienté la campagne du NPD sur diverses autres avenues, avec de moins en moins d'agressivité sur ce qui s'avérait être l'enjeu numéro un de la campagne ${ }^{18}$. À l'opposé, les libéraux ont fait de la question du libre-échange leur cheval de bataille. Ce faisant, peu à peu, le leadership de la lutte contre le libre-échange est passé du côté libéral. Au plan du discours, les libéraux ont littéralement doublé le NPD sur sa gauche, alors que le message néodémocrate manquait de piquant et de force de conviction. Cette réalité, perceptible en Ontario et au Manitoba, l'était aussi au Québec. Conséquemment, de manière globale, alors qu'il devançait les libéraux en début de campagne, le NPD a progressivement vu une part de ses appuis lui glisser des mains.

C'est là, croyons-nous, un des principaux facteurs explicatifs des faibles résultats du NPD au plan canadien. Le fait d'avoir cédé le pas aux libéraux sur la question du libre-échange a vraisemblablement empêché le NPD de renforcer ses positions de manière significative, à l'exception de deux provinces où sa présence a été plus importante, et où les libéraux ne représentent pas une force menaçante. Mais ces seules considérations ne suffisent pas lorsqu'il s'agit de mettre en lumière le nouveau revers essuyé par le NPD au Québec, plus sérieux celui-là.

18. En l'occurrence, la performance d'Ed Broadbent sur cet enjeu aux deux débats des chefs, notamment celui en anglais, s'est avérée très peu convaincante. À un certain moment, le chef du NPD ne semblait même plus être de la partie. 


\section{L'épreuve du Québec}

L'analyse du sort fait au NPD au Québec le 21 novembre 1988 ne peut être menée à bien sans d'abord rendre compte de manière plus détaillée de ses performances dans les 75 comtés de la province.

Tableau 2: Les élections fédérales du 21 novembre 1988. Tableau des quinze meilleurs résultats du NPD au Québec ${ }^{1}$

\begin{tabular}{lcr}
\hline $\begin{array}{l}\text { Circonscription } \\
\text { (candidat-e) }\end{array}$ & $\begin{array}{c}\text { \% des voix } \\
\text { exprimées en }\end{array}$ & $\begin{array}{r}\% \text { du NPD } \\
\text { en 1984 }\end{array}$ \\
\hline Témiscamingue (Rémy Trudel) & 37,8 & 5,4 \\
Chambly (Phil Edmonston) & 31,4 & 11,0 \\
Saint-Maurice (Claude Rompré) & 30,1 & 3,5 \\
Abitibi (Gerry Lemoyne) & 26,4 & 7,9 \\
Laurier-Sainte-Marie (François Beaulne) & 22,0 & $*$ \\
Jonquière (Françoise Gauthier) & 20,9 & 5,5 \\
Hochelaga-Maisonneuve (Gaétan Nadeau) & 20,5 & 10,9 \\
Outremont (Louise O'Neil) & 20,4 & 18,6 \\
Rosemont (Giuseppe Sciortino) & 20,2 & 10,6 \\
Langelier (Pauline Gingras) & 20,0 & 11,5 \\
Lévis (Jean-Paul Harney) & 19,4 & 18,4 \\
Champlain (Jean Crête) & 19,2 & 6,7 \\
Lac-Saint-Jean (Jean Paradis) & 18,4 & $* *$ \\
Mercier (André Cordeau) & 18,3 & 8,9 \\
Saint-Hubert (Nicole Desranleau) & 18,0 & $*$ \\
& & \\
\hline
\end{tabular}

(1) Les résultats ne tiennent pas compte des abstentions et des votes rejetés.

* Nouvelle circonscription en 1988.

** Dans ce comté, le NPD a recueilli $5,1 \%$ des voix en 1984 . A l'élection partielle de juin 1988, Jean Paradis a obtenu $9,6 \%$ des suffrages exprimés.

Sources: Pour 1984, Drouilly Pierre, Statistiques électorales fédérales du Québec, 1867-1965, VLB Éditeur, 1986. Pour 1988, Données préliminaires du Directeur général des élections.

Au Québec, le NPD a recueilli $14 \%$ des voix exprimées, et aucun de ses 75 candidats n'a réussi à se faire élire. En ce qui concerne 
les suffrages exprimés, le NPD a bel et bien bénéficié, en comparaison avec le passé, d'une progression de son support électoral. Le vote a dépassé le score de 1965 (12\%), établi sous le leadership de Robert Cliche. Trois candidats du NPD au Québec ont recueilli plus de 10\% des voix exprimées dans leur comté. Il s'agit de Rémy Trudel dans le comté de Témiscamingue (avec 37,8\% des voix), de Phil Edmonston dans le comté de Chambly (avec $31,4 \%$ des voix) et de Claude Rompré dans le comté de Saint-Maurice (avec 30,1\% des voix). Dans ces trois circonscriptions, le NPD s'est classé au deuxième rang. Il en a fait de même dans quatre autres comtés (Abitibi, Champlain, Jonquière et Lac-Saint-Jean). Par ailleurs, le NPD a été en mesure de dépasser le seuil des $15 \%$ des voix dans 25 comtés.

L'examen des résultats du NPD dans les 15 circonscriptions où ce parti a obtenu ses meilleurs résultats (en pourcentage des voix exprimées) nous permet d'indiquer le plus important changement qualitatif survenu pour le NPD au Québec.

Contrairement aux élections fédérales passées, le NPD a connu une certaine progression de son vote parmi la population francophone, tant dans la région de Montréal que dans les circonstriptions situées en province. Ces gains ont été surtout arrachés aux libéraux. Les exemples de Jonquière, de Mercier ou de Rosemont sont, à cet égard, révélateurs de la croissance des appuis du NPD en milieu francophone. C'est ce changement en direction des francophones qui explique fondamentalement le fait que le NPD, au Québec, a bénéficié de 14\% des voix exprimées en 1988 contre 8,8\% en 1984, soit une hausse de $5,2 \%$ de son support global. Quant aux comtés à majorité ou à forte minorité anglophone ou allophone, le NPD n'y a pas connu de renforcement notoire. Ainsi, dans le comté d'Outremont, le NPD est passé de 18,6\% des voix exprimées en 1984 à 20,5\% en 1988 (la progression des voix étant obtenue surtout dans le bas-Outremont et parmi les francophones); dans le comté de Saint-Henri-Westmount, les appuis du NPD sont passés de 14,5\% à 15,6\%; dans LaSalle-Émard, de $9,4 \%$ à $10,8 \%$. Dans d'autres cas, au contraire, le NPD a même subi une baisse de ses appuis. C'est le cas dans le comté de Lachine: les voix du NPD y ont diminué de $11,9 \%$ en 1984 à 7,9\% en 1988. Dans Notre-Dame-de-Grâce, phénomène semblable: les voix du NPD y sont passées de 13,9\% en 1984 à 12,3\% en 1988. Ces derniers reculs du 
NPD-Québec chez les anglophones et les allophones, ont été doublés d'un renforcement des votes en faveur de libéraux. Sont-ils liés aux ressentiments de ces communautés face à l'Accord du Lac Meech soutenu par l'aile fédérale du parti, ou face à l'orientation plus nationaliste du NPD-Québec? Il est permis de le croire.

Par ailleurs, le NPD a connu un renforcement dans les circonscriptions urbaines à base populaire et ouvrière, comme Hochelaga-Maisonneuve ou Laurier-Sainte-Marie. Là où la présence des organisations syndicales est plus forte et ressentie, la performance du NPD a aussi connu un certain renforcement (Abitibi, Jonquière, Langelier, etc.).

Même si le NPD a connu un relatif affermissement de ses appuis chez les Québécois francophones, il faut bien situer ces progrès dans leurs justes proportions. Il ne s'agit que d'une légère progression, puisque la très grande majorité du vote chez les francophones s'est exprimée en faveur des conservateurs. Par contre, le Parti conservateur semble trouver ses appuis les plus importants chez les classes moyennes et les classes les mieux nanties. Dans les milieux francophones défavorisés, le Parti conservateur a aussi recueilli une part de ses appuis, mais là le taux d'abstention s'est avéré élevé et le NPD est parvenu à y gagner une partie du vote ${ }^{19}$.

En résumé, le NPD a renforcé quelque peu ses appuis au Québec, en particulier chez les francophones et les classes populaires. Par contre, cette progression s'est avérée très limitée et le NPD n'a finalement fait élire aucun candidat. Ainsi, malgré les gains réalisés, le NPD n'est pas encore sorti de l'impasse dans laquelle il est enfermé au Québec depuis sa fondation.

Mais il y a plus. Compte tenu que le NPD escomptait effectuer une percée significative au Québec en 1988 et, pour la première fois de son histoire, y faire élire un ou des députés, le résultat représente un revers de taille. Il s'agit d'un échec en regard de l'occasion qui lui était offerte, une occasion comme il ne s'en est pas présenté depuis sa fondation. Après 1961, en effet, le NPD n'a jamais connu de

19. À ce propos, voir Boily, Pelletier et Serré, Le Devoir des 8 et 9 décembre 1988. 
circonstances aussi favorables que celles qui ont prévalu dans les deux années qui ont précédé le scrutin général. Reposant sur les reculs subis par les libéraux et la chute de popularité des conservateurs minés par les scandales, la nouvelle situation offrait une ouverture sans précédent pour le NPD. Celle-ci, en 1987 et 1988, lui permettait de renforcer ses appuis et même d'escompter l'élection de candidats au Québec. Mais le NPD n'a pas su en tirer profit.

Partant de ces constats, il est possible de regrouper sous quelques volets les facteurs qui rendent compte de la faiblesse du NPD au Québec dans cette élection du 21 novembre 1988.

Dans un premier temps, dans la bataille du libre-échange, le NPD a, bien sûr, fait face au Québec à une situation bien particulière. La solide coalition en faveur du libre-échange qui s'y est établie était sans égale dans le reste du Canada. Ce fort courant, soutenu par les deux grands partis de l'Assemblée nationale du Québec, ne pouvait qu'ajouter aux difficultés du NPD, d'autant plus que les appuis recueillis par le gouvernement Bourassa dans les sondages restaient toujours élevés. Mais par ailleurs, dans la bataille contre le libreéchange, il appert que les libéraux, au Québec comme dans le reste du Canada, ont pris l'ascendant sur le NPD. Comme nous l'avons déjà souligné, la timidité de l'intervention du NPD et le manque de force de conviction de ses candidats ont permis aussi aux libéraux du Québec, comme au Manitoba ou en Ontario, de se présenter comme les porteparole de l'oppositin au libre-échange. Les Lapierre, les Malépart et les Garneau (ce dernier ayant été battu de justesse) ont joué à fond la carte populiste et, jusqu'à un certain point, ont déjoué le NPD sur ce terrain. La campagne néo-démocrate, pour sa part, est restée centrée sur Ed Broadbent et sur un discours tiédi, sans relief et sans éclat. Les libéraux, se présentant comme la force d'opposition au libre-échange et concentrant leurs interventions publiques sur cet enjeu, ont ainsi érodé une part des appuis potentiels du NPD. Il en va de même en ce qui concerne la série de scandales qui ont frappé le gouvernement Mulroney de 1984 à 1988 . Le NPD n'a aucunement utilisé à son profit cette situation, laquelle avait été une des principales sources d'insatisfaction au Québec. Ce sont les libéraux qui se sont approprié l'enjeu, avec une campagne d'intervention publique agressive et 
percutante. La stratégie néo-démocrate, axée sur la modération et l'image de son chef, a donc fait preuve de peu de mordant au Québec.

D'autre part, compte tenu de la spécificité du Québec, le NPD ne pouvait y effectuer une percée significative qu'à condition de «coller» à la réalité québécoise et de souscrire pleinement aux droits nationaux des Québécois. Cette condition était d'autant plus évidente que Brian Mulroney et son équipe comptaient sur le nationalisme québécois. Or, à ce propos, plusieurs éléments ont contribué à affaiblir les efforts du NPD et à en faire un parti perdant au Québec.

Tout d'abord, au-delà de sa popularité personnelle soulignée dans les sondages en 1987 et 1988, Ed Broadbent, comme chef de parti, n'a pas été en mesure de conquérir le Québec. Ontarien et anglophone, se comparant lui-même à un «étranger» au Québec ${ }^{20}$, éprouvant de sérieuses difficultés à s'exprimer en français, le chef du NPD n'a pas su gagner les Québécois. Sur ce plan, Brian Mulroney possédait un net avantage. À cet égard, doit-on tirer de la victoire du chef conservateur un enseignement de portée plus générale? La question se pose. Bien qu'elle ne soit pas le propos central de notre article, elle mérite d'être soulignée. Depuis 20 ans, en effet, la réalité de la question nationale a donné lieu à un fait indiscutable: même à l'occasion de la victoire de Joe clark en 1979, les Québécois ont toujours concédé la majorité de leurs voix à un parti fédéral dont le chef est d'origine québécoise et francophone. Par ce biais, une bonne part des Québécois ont cru y déceler une meilleure défense de leurs intérêts. Cette perception a été abondamment utilisée par Pierre-Elliott Trudeau au cours des années 1960 et 1970 . Ne s'est-il pas présenté, à grand renfort de déclarations, comme l'incarnation du «french power» à Ottawa. En tout état de cause, cette stratégie a servi les libéraux pour un temps, du moins jusqu'aux élections fédérales de 1980, qui ont précédé le référendum québécois et le coup de force constitutionnel de

20. Commentaire fait le 10 novembre 1988 , en relation avec la polémique entourant les divisions au sein du NPD-Québec au sujet de la question linguistique. 
1982. En 1984 et 1988, cette même stratégie a aussi été utilisée par Brian Mulroney. Elle lui a profité et a désavantagé Ed Broadbent ${ }^{21}$.

D'autre part, outre l'handicap du chef, il est apparu de manière plus évidente, à l'approche du 21 novembre, que la direction fédérale du NPD n'avait pas fondamentalement changé ses positions sur la question nationale québécoise. Les timides ouvertures de 1987 ont rapidement fait place aux tergiversations et aux interrogations. $\grave{A}$ quelques jours du scrutin, le chef fédéral a réitéré ses positions centralisatrices et son adhésion aux principes de la Loi sur les langues officielles. Par la même occasion, il a refusé de se prononcer clairement en faveur de la loi 101. À l'opposé, sept candidats du NPDQuébec, dont Jean-Paul Harney, se sont prononcés contre les possibles intrusions que la dite loi fédérale pourrait permettre contre la loi 101 par le biais de programmes fédéraux de promotion de l'anglais au Québec. Ils se sont également prononcés en faveur du recours à la clause «nonobstant» de manière à assurer la primauté des droits collectifs des Québécois sur la Charte canadienne des droits et libertés. D'autres candidats anglophones du NPD au Québec 22 , secondés par le journal The Gazette, ont protesté contre cette prise de position des sept. Touché par la controverse, le chef fédéral s'est finalement dissocié de la position des candidats partisans de la primauté et de la défense de la loi 101. Ces divergences, manifestées à la veille du scrutin, ont vraisemblablement contribué à la baisse des appuis du NPD parmi la minorité anglophone du Québec. Mais elles ont aussi nourri des interrogations supplémentaires parmi les Québécois francophones, dans un contexte électoral où, contrairement aux présomptions des dirigeants fédéraux du parti, les aspirations nationales du peuple québécois n'étaient aucunement dissipées. Cette situation n'a pu que profiter à Brian Mulroney, qui utilisait au profit des conservateurs le nationalisme québécois.

21. Cette dimension, même si elle peut difficilement échapper aux stratèges néo-démocrates, n'a pourtant pas été retenue par ceux-ci lorsqu'Ed Broadbent, en mars 1989, a finalement annoncé sa démission comme chef du parti et que s'est ouverte la campagne au leadership de 1989.

22. Dont Maria Peluso, candidate dans Notre-Dame-de-Grâce. 
En liaison avec ces principaux facteurs mentionnés, le NPD a connu d'autres difficultés qui ont contribué à sa faible performance. $\mathrm{La}$ campagne électorale a été pilotée et centralisée à Ottawa, dans l'entourage immédiat d'Ed Broadbent, sans représentation québécoise significative $^{23}$. Aussi, la structure unique du NPD, masquant mal une division évidente sur des aspects fondamentaux de son programme, ne pouvait qu'affaiblir les efforts de construction, semer la confusion et miner ainsi le support électoral ${ }^{24}$. Ces facteurs, sans contredire les premiers, fournissent des éléments complémentaires d'explication de la ratée du NPD.

\section{Conclusion}

À quelques nuances près, le résultat de 1988 a confirmé l'héritage du NPD sur la scène fédérale. Il l'a même renforcé. Les forces et les députés du NPD sont encore davantage confinés dans l'Ouest canadien et, dans une moindre mesure, en Ontario. Dans les Maritimes, le NPD est quasiment évincé du paysage politique. Au Québec, le NPD a, bien sûr, augmenté ses appuis électoraux. Le support électoral a légèrement augmenté. Mais aucun député n'a pu être élu, affaiblissant d'autant l'impulsion qui a été donnée en 1987 et 1988 et les perspectives de construction du NPD sur la scène électorale québécoise. En témoigne l'évolution du membership qui s'était accru en 1987 et 1988. Après l'élection du 21 novembre 1988, celui-ci s'est affaissé considérablement, comme l'a dévoilé le congrès du NPDQuébec d'avril 1989. Tout compte fait, à l'Est des Grands Lacs, la percée qu'escomptait effectuer le NPD n'a jamais pris forme.

23. À ce sujet voir le point de vue de Pierre Graveline, coprésident du NPD-Québec, dans la déclaration intitulée «Ed Broadbent et le Québec: le rendez-vous manqué», publié dans Le Devoir du ler décembre 1988.

24. À son congrès d'avril 1989, le NPD-Québec a finalement remis en cause cette structure unique et a convenu de se doter de deux ailes distinctes. 
En regard du Québec, nous constatons que le présumé «virage historique» effectué par le NPD fédéral en 1987 n'a guère dépassé les grandes proclamations verbales. Pendant la campagne électorale, le parti fédéral a réitéré ses traditionnelles positions centralisatrices et a refusé de souscrire aux aspirations d'une très large portion de la population québécoise sur le plan linguistique. Les prises de positions post-électorales du NPD, en 1988 et 1989, confirment cette allégation. La direction du parti, Ed Broadbent en tête, a condamné le recours à la clause «nonobstant» par le gouvernement Bourassa pour soustraire le Québec du jugement de la Cour suprême qui déclarait inconstitutionnelles les prescriptions de la loi 101 en matière d'affichage. D'autre part, la croisade menée par les dirigeants néodémocrates de l'Ouest canadien contre l'Accord du Lac Meech, notamment leur opposition à la reconnaissance du Québec comme société distincte, est une autre illustration du dérapage du NPD fédéral sur la question du Québec. Près de 30 ans après sa fondation, le nouvel échec de 1988 et ses retombées, témoignent en particulier de ce que la social-démocratie au Canada n'a jamais su et n'a jamais voulu véritablement intégrer la question nationale québécoise à son développement. Les dirigeants du parti ont toujours refusé de reconnaître et de souscrire aux aspirations nationales du peuple québécois, n'y voyant que des dangers et des menaces à l'unité canadienne. Ils ont toujours fait obstacle, dans les rangs du NPD, à une réelle prise en charge de cette dimension fondamentale de la vie politique du Québec et du Canada. La direction du NPD a constamment réitéré, en 1988 comme par le passé, ses positions ultra-fédéralistes et centralisatrices, minant par le fait même les nouveaux appuis recherchés au Québec. En Grande-Bretagne, il est de notoriété que le Parti travailliste s'est continuellement embourbé dans la question nationale irlandaise. Au Canada, nous pouvons certainement établir un certain parallèle. Malgré la différence de situation, le NPD s'est aussi empêtré dans le cheminement québécois qu'il n'a jamais su comprendre et assumer. Le peuple québécois, dans sa très grande majorité, a démontré qu'il ne se reconnaît pas encore dans ce parti, après ses 30 ans d'existence. Le NPD n'a donc pas pu saisir une des rares occasions de son histoire. Conséquemment, l'avenir immédiat n'augure rien de 
L'ÉLECTION FÉDÉRALE DE 1988 ET LA DÉFAILLANCE DU NPD 103

très éclatant pour le NPD au Québec, ni pour le parti fédéral, ni pour son aile québécoise. 